JOURNAL OF

APPLIED CRYSTALLOGRAPHY

ISSN 1600-5767

Received 28 February 2017

Accepted 25 April 2017

Edited by G. Renaud, CEA-Grenoble DSM/ INAC/SP2M/NRS, Grenoble, France

Keywords: water; graphene oxide; WAXS; wide-angle $\mathrm{X}$-ray scattering; $\mathrm{X}$-ray scattering modelling.

Supporting information: this article has supporting information at journals.iucr.org/j

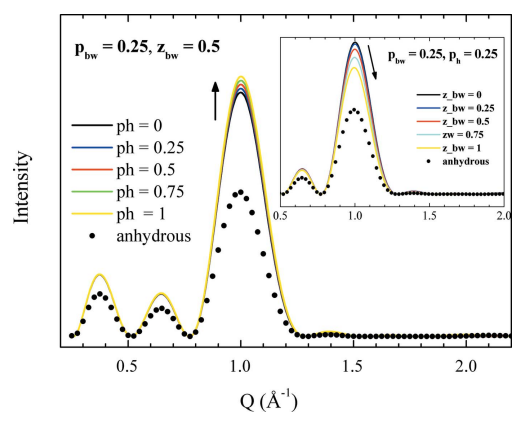

C 2017 International Union of Crystallography

\section{Intercalated water in multi-layered graphene oxide paper: an X-ray scattering study}

\author{
Stéphan Rouzière, ${ }^{\mathrm{a} *}$ J. David Núñez, ${ }^{\mathrm{b}}$ Erwan Paineau, ${ }^{\mathrm{a}}$ Ana M. Benito, \\ Wolfgang K. Maser ${ }^{b}$ and Pascale Launois ${ }^{a}$
}

\author{
aLaboratoire de Physique des Solides, CNRS, Université Paris-Sud, Université Paris-Saclay, Orsay Cedex, 91405, France, \\ and ${ }^{\mathbf{b}}$ Instituto de Carboquimina (ICB-CSIC), Zaragoza, E-50018, Spain. *Correspondence e-mail: \\ stephan.rouziere@u-psud.fr
}

X-ray scattering (XRS) experiments have been performed on multi-layered graphene oxide (GO) paper. GO can be easily hydrated as water naturally intercalates in the hydrophilic nanostructure. The effect of the intercalated water on the XRS signals is measured during dehydration under thermal treatment in the temperature range $298-473 \mathrm{~K}$ as well as during hydration under saturated water vapour pressure. A simple modelling of the XRS signals by taking into account the presence and the type of intercalated water (bound water or physisorbed water) is introduced. It allows an explanation of the variations of intensity and position of XRS reflections observed experimentally.

\section{Introduction}

Water confined in nanometric carbon structures can exhibit structural and dynamical properties different from its bulk properties. For instance, an ultra-fast water transport phenomenon has been observed in membranes based on carbon nanotubes (Majumder et al., 2005; Holt et al., 2006; Guo et al., 2015) and in graphene-based membranes (Nair et al., 2012), opening the way to potential applications in nanofluidics. Precise molecular sieving through graphene oxide membranes has been highlighted (Joshi et al., 2014). Nanoconfined water can also form various kinds of ice in onedimensional carbon nanotubes (Kolesnikov et al., 2004; Kyakuno et al., 2011; Mochizuki \& Koga, 2015) or within twodimensional graphene assemblies (Algara-Siller et al., 2015; Li et al., 2015). Among carbon nanostructures, graphene oxide (GO), referring here to multilayered stacks of individual graphene oxide sheets, is highly hydrophilic, in contrast with the more hydrophobic carbon nanotubes or pristine graphene. Therefore, GO can easily trap water with interesting hydration and solvation properties (Talyzin et al., 2014). It can be considered as a two-dimensional model system for the study of water confined between hydrophilic nanosheets.

Although the exact chemistry and structure of GO is still under debate (Dreyer et al., 2010; Loh et al., 2010), the most accepted structural model (Lerf et al., 1998) describes GO as a non-stoichiometric material consisting of oxidized graphene sheets with a random arrangement of oxygen functional groups (OFGs), namely, epoxide and hydroxyl groups located on the graphene sheet's basal plane and carbonyl or carboxyl groups at the sheet edges, together with residual non-functionalized graphitic areas. The oxidation process is responsible for the expansion of the interlayer distance between graphene sheets, from about $3.4 \AA$ in turbostratic graphite to $\sim 6 \AA$ in GO. Adsorption of water through hydration can expand 
further the interlayer distance up to $\sim 12 \AA$ (Lerf et al., 2006; Buchsteiner et al., 2006; Barroso-Bujans et al., 2010; Rezania et al., 2014; Talyzin et al., 2014), its value being related to the water content. The thermal reduction of GO, which allows tuning of its opto-electronic properties, induces the removal of water as well as OFGs with a partial restoration of $s p^{2}$ carbon bonds, and also the removal of carbon atoms from the GO plane, introducing new structural vacancies throughout the graphene sheets (Gao et al., 2009; Pei \& Cheng, 2012). It has been suggested that water may play a significant role in the irreversible decomposition of OFGs during the reduction process of GO under thermal treatment (Acik et al., 2010; Núñez, 2015). Therefore, study of the intercalated water in $\mathrm{GO}$ as a function of temperature can provide valuable information for the understanding of the thermal reduction process of GO.

Dynamical properties of water confined in GO have been studied by neutron scattering (Buchsteiner et al., 2006; Lerf et al., 2006) and by infrared and broadband dielectric spectroscopies (Cerveny et al., 2010). These studies indicate the presence of two types of intercalated water with different translational and rotational mobilities, namely (i) bound water, related to molecules strongly bound to OFGs (by hydrogen bonding), and (ii) physisorbed water, representing water molecules physically adsorbed between GO layers. The structural characterization of water uptake by GO layers has been mainly assessed by X-ray diffraction with samples in the form of powder or paper exposed to water vapour or soaked in liquid water (Lerf et al., 2006; Talyzin et al., 2011; You et al., 2013; Talyzin et al., 2014). The nature of the intercalated water was ascertained by considering the interlayer distance $d_{001}$ and its variation upon sorption/desorption cycling, during which physisorbed water can be reversibly removed by vacuum or temperature treatment, essentially below $T=373 \mathrm{~K}$ (BarrosoBujans et al., 2010; Talyzin et al., 2011; Zhu et al., 2012). Conversely, the removal of bound water takes place at higher temperatures and implies the concomitant irreversible decomposition of OFGs (Huh, 2011; Pan et al., 2016). Considering the size of a water molecule (mean van der Waals diameter $\sim 2.8 \AA$ ), the gradual expansion of $d_{001}$ up to $\sim 8 \AA$ has been ascribed to a continuous process of incorporation of water molecules into specific sites within GO layers such as voids between OFGs or defects in graphene sheets, while larger values of $d_{001}$ above $\sim 9 \AA$ have suggested the formation of a water monolayer between the hydrated GO layers (Buchsteiner et al., 2006; Lerf et al., 2006; Talyzin et al., 2011; Rezania et al., 2014). All previous studies have focused essentially on the variations of position of the 001 reflection, but to the best of our knowledge, no X-ray scattering modelling has been proposed to understand the variations of intensity during hydration of GO layers. Such modelling will allow us to describe not only the GO structure overall but also how the location of intercalated water inside the structure can explain the variations of position and intensity of $00 \mathrm{~L}$ reflections observed experimentally.

In the present work, we aim to investigate the effect of intercalated water in GO on X-ray scattering (XRS) data. The experiments are carried out under vacuum and with a largesurface detector, which allows all the scattering features of GO paper to be observed. The effect of intercalated water on XRS signals is assessed in experiments performed under thermal treatment for the dehydration process or under saturated water vapour pressure for the hydration process. Modelling of the GO structure with intercalated water, either bound water or physisorbed water, is introduced as an efficient tool for XRS analysis and interpretation.

\section{Experimental method}

First, graphite oxide was prepared from graphite flakes using a modification of Hummers' method, as reported elsewhere (Hummers \& Offeman, 1958; Vallés et al., 2012). Second, the resulting graphite oxide was dispersed in distilled water, ultrasonicated and centrifuged, resulting in a light-browncoloured dispersion of exfoliated graphene oxide. Third, freestanding flexible GO papers were prepared by vacuumassisted flow filtration of $500 \mathrm{ml}$ of the aqueous GO, with a concentration of $0.15 \mathrm{mg} \mathrm{ml}^{-1}$. The paper assemblies were rinsed with water and dried overnight before being peeled off from the filter. The physical-chemical properties of the GO paper were determined by thermogravimetric analyses (TGA) and X-ray photoelectron spectroscopy (XPS) (see supporting information). Pieces of GO paper of size $\sim 4 \times 2 \mathrm{~mm}$ were used for XRS measurements.

XRS measurements were carried out either in air or under primary vacuum, or else under saturated water vapour pressure, with molybdenum radiation $(\lambda=0.711 \AA)$ delivered by a multilayer mirror optics (Xenocs FOX 2D Mo 25-INF) coupled with a rotating anode generator (Rigaku HU3R, $40 \mathrm{kV}-40 \mathrm{~mA}$ ). Experiments under vacuum (the sample is placed in an evacuated chamber) allowed a high signal over background ratio, beneficial for measuring weak XRS signals. XRS images were recorded by an X-ray-sensitive plate detector (MAR345) placed after the exit window of the chamber.

Thermal treatment of the GO paper was performed with a purpose-built vacuum-compatible $\mathrm{X}$-ray resistance furnace which consists of a copper block heated by a resistor with entrance and exit windows for the X-ray paths. GO paper is glued with ceramic paste on a copper sample holder to be introduced inside the block. A thermocouple is inserted into the sample holder, close to the sample, in order to control the temperature. The temperature ramp rate was $\sim 2 \mathrm{~K} \mathrm{~min}^{-1}$. The acquisition time of an XRS image was $30 \mathrm{~min}$ at each temperature stage.

Hydration was carried out by placing a reservoir of distilled water in the chamber, which was pumped until equilibrium in order to reach the value of saturated vapour pressure $(23.8 \mathrm{mbar}$ at $298 \mathrm{~K} ; 1 \mathrm{mbar}=0.1 \mathrm{kPa})$.

XRS measurements were performed in transmission geometry on GO papers oriented parallel to the incident X-ray beam. The XRS technique applied allows measurement of the anisotropic signals on the large-area detector, which gives information on orientational features of the GO 
structure. Image processing with intensity extraction was achieved with in-house-developed software.

\section{Experimental results}

\subsection{X-ray scattering from GO paper}

A typical XRS image of GO paper obtained at room temperature and under primary vacuum is shown in Fig. 1 . The method applied allows the respective contributions from the in-plane and out-of-plane structural characteristic features of the GO paper to be observed. The preferential orientation of periodic stacked GO layers is clearly shown by the angular anisotropy of the $00 \mathrm{~L}$ reflections. For the GO paper under study, two 001 reflections are observed. The first one, located at scattering wavevector magnitude $Q \simeq 0.95 \AA^{-1}$, corresponds to the interlayer distance $d_{001}\left(=2 \pi / Q_{001} \simeq 6.6 \AA\right)$ between stacked GO sheets covered by OFGs. No higherorder $00 L$ reflections are observed. A second peak labelled $001_{\text {gr }}$, located at $Q \simeq 1.78 \AA^{-1}$, refers to regions with graphitic character $\left(d \simeq 3.5 \AA\right.$ ). The second-order $002_{\mathrm{gr}}$ reflection is hardly visible (see inset of Fig. 1). The $00 L_{\mathrm{gr}}$ reflections essentially correspond to remaining impurities arising from periodic stacking of non-oxidized graphitic sheets.

XRS features characteristic of the in-plane hexagonal arrangement of carbon atoms within a graphene sheet are illustrated by the 10 and 11 reflections, with their intensity

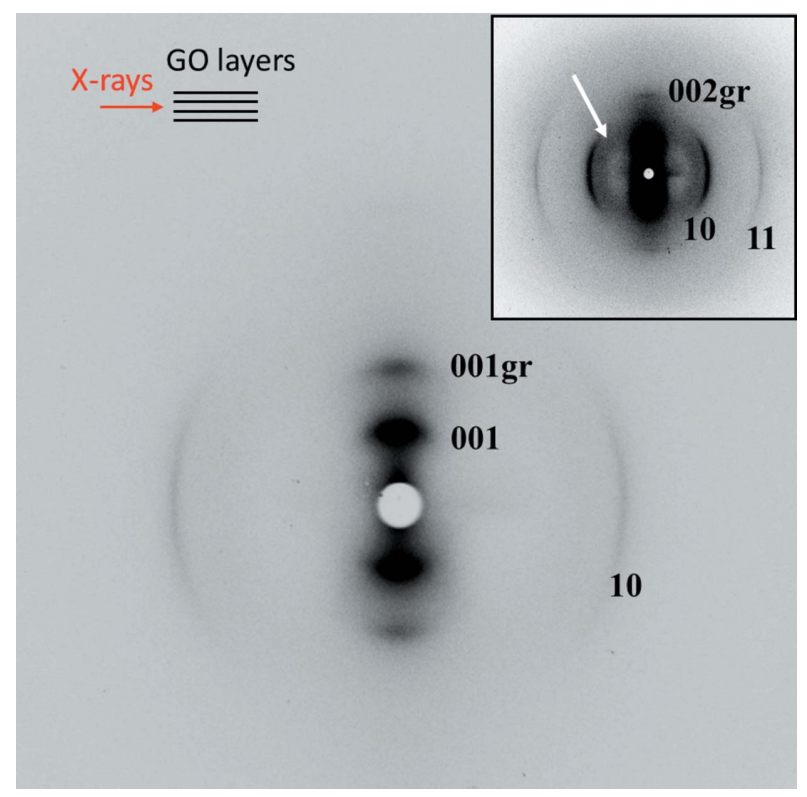

Figure 1

XRS image obtained under vacuum and at room temperature on a GO paper. In the inset, the saturation level was divided by a factor of ten, to bring out the weakest scattering signals. Indexed peaks correspond to the 001 reflection of the periodic stacking of GO layers, and 001 (labelled $001_{\mathrm{gr}}$ ) and 002 (labelled $002_{\mathrm{gr}}$ ) of the periodic stacking of graphitic sheets, while the 10 and 11 reflections arise from the in-plane contribution of both GO and graphitic sheets. Isotropic broad diffuse scattering is visible, indicated by the white arrow. XRS measurements were performed in transmission geometry on GO paper with GO layers oriented parallel to the incident X-ray beam, as sketched in the upper left corner of the figure. maximum at $90^{\circ}$ from the $00 L$ reflections. Both $\mathrm{GO}$ and graphitic sheets contribute to the intensities of $H K$ peaks and they cannot be discriminated owing to the lack of experimental resolution. The measured position roughly corresponds to the expected lattice parameter value $d_{10}=2.46 \AA$ and the $\mathrm{C}-\mathrm{C}$ bond length $\left(d_{10} / 3^{1 / 2}\right)$ of $1.42 \AA$ of a graphene sheet. The absence of $H K L$ reflections (with $H$ or $K$ and $L$ having nonzero values) indicates that neither GO nor graphite stacks exhibit orientational and/or positional correlations between the sheets.

Under vacuum conditions, a weak isotropic diffuse scattering signal can be measured (see inset in Fig. 1) at wavevector magnitudes $Q$ ranging from 2 to $2.7 \AA^{-1}$ (Fig. $2 b$ ). This signal most likely refers to the random orientation distribution of oxygen functional, carbonyl and carboxyl groups, located at the edges of the GO sheets. Water bound to these OFGs contributes to this weak scattering signal likewise.

\subsection{Thermal treatment of GO paper}

XRS images similar to Fig. 1 were recorded during in situ thermal treatment of a GO paper in the temperature range [298-473 K]. Intensity scans along the vertical and horizontal directions, corresponding to $00 L$ and $H K$ intensity maxima, respectively, were performed on each image. Fig. 2(a) shows the evolution of $00 \mathrm{~L}$ reflections as a function of temperature. The position of the $001 \mathrm{GO}$ reflection moves continuously towards larger $Q$ values, corresponding to a variation of the interlayer distance $d_{001}$ from 6.6 to $5.5 \AA$, while its intensity decreases. Conversely, the position and intensity of the graphitic $001_{\mathrm{gr}}$ reflection do not vary noticeably. The evolution of the integrated intensities (peak area) of the $001,001_{\mathrm{gr}}$ and 10 reflections is plotted in Fig. 2(c). While the $001_{\mathrm{gr}}$ peak remains unaffected by the thermal treatment, the 001 reflection's integrated intensity is reduced by $60 \%$ at $T=473 \mathrm{~K}$, which indicates a strong chemical modification of the GO layers. Furthermore, an acceleration of the thermal process of chemical decomposition of GO layers is observed, with a steeper slope in the decrease of the intensity above $423 \mathrm{~K}$. Likewise, the integrated intensity of the 10 reflection decreases above $423 \mathrm{~K}$, indicating the deterioration of the graphene sheets of the GO layers with the formation of defects or holes (Pan et al., 2016). Note that the weak isotropic diffuse scattering is strongly reduced in the same temperature range [423-473 K] before it completely vanishes at $473 \mathrm{~K}$ (Fig. 2b). We had tentatively ascribed this weak isotropic scattering to the random orientation distribution of OFGs (carbonyl and carboxyl groups) with water molecules bound to them and located at the edges of the GO sheets. Its disappearance is additional evidence of the strong modification of the chemical composition of GO layers. In short, the thermal treatment of GO is responsible for the removal of OFGs, both in the plane and at the edges of the graphene-like sheets.

The dependence of the interlayer distance between GO layers, $d_{001}=2 \pi / Q_{001}$, on temperature is plotted in Fig. $2(d)$ for measurements under vacuum and at ambient pressure. As 
mentioned before, $d_{001}$ is related to the periodic stacking of GO layers with intercalated molecules, OFGs (epoxide and hydroxyl groups) and water, between them. In the range [298$373 \mathrm{~K}$ ], the $d_{001}$ value obtained at ambient pressure evolves from $7.5 \AA$ (room temperature) down to $6.4 \AA(T=373 \mathrm{~K})$, while $d_{001}$ remains roughly constant under vacuum $\left(d_{001} \simeq\right.$ $6.4 \AA$ ). This behaviour is ascribed to the removal of physisorbed water molecules obtained either by mild heating or by the evacuation process. Above $T=373 \mathrm{~K}$, the interlayer distance gradually decreases towards $5.5 \AA$ at $T=473 \mathrm{~K}$. A steeper slope is clearly observed above $423 \mathrm{~K}$, which points towards the accelerated degradation of GO layers through the removal of OFGs and bound water. This degradation process can also present some kinetic aspect at a given temperature stage. To assess such effects, additional measurements were performed after $12 \mathrm{~h}$ at $473 \mathrm{~K}$, and these show a further decrease of the interlayer distance from 5.5 to $4.5 \AA$ [see bottom inset in Fig. $2(d)]$. Heating to $T=573 \mathrm{~K}$ induces a further decrease of the interlayer distance down to $4 \AA$. The interlayer distance would eventually reach the value of the turbostratic graphite structure $\left(d_{001} \simeq 3.4 \AA\right)$ at higher temperatures when all OFGs have been removed, as already demonstrated (Huh, 2011). These XRS observations are in agreement with the physical-chemical characterization of the GO papers. Indeed, XPS and TGA results (see supporting information) confirm the removal of OFGs and bound water (Stankovich et al., 2007; Núñez, 2015; Núñez et al., 2017) in the temperature range between 403 and $533 \mathrm{~K}$.

Evolution of the $Q$ width of the 001 reflection as a function of temperature is reported in the top inset of Fig. 2(d). Up to $T=373 \mathrm{~K}$ and under vacuum, the 001 reflection is located at $Q_{001} \simeq 0.98 \AA^{-1}$ with an FWHM close to $0.25 \AA^{-1}$. The FWHM is slightly smaller for measurements in air than for those under vacuum until it reaches the same value at $373 \mathrm{~K}$. At higher temperatures, the FWHM slightly increases, but it rises steeply above $423 \mathrm{~K}$ owing to the accelerated chemical deterioration of the GO layer ( $\mathrm{FWHM}=0.35 \AA^{-1}$ at $473 \mathrm{~K}$ ), whereas the angular width of the 001 reflection does not change significantly (see XRS images at 373 and $473 \mathrm{~K}$ in Fig. S3). On the other hand, the position and FWHM of the
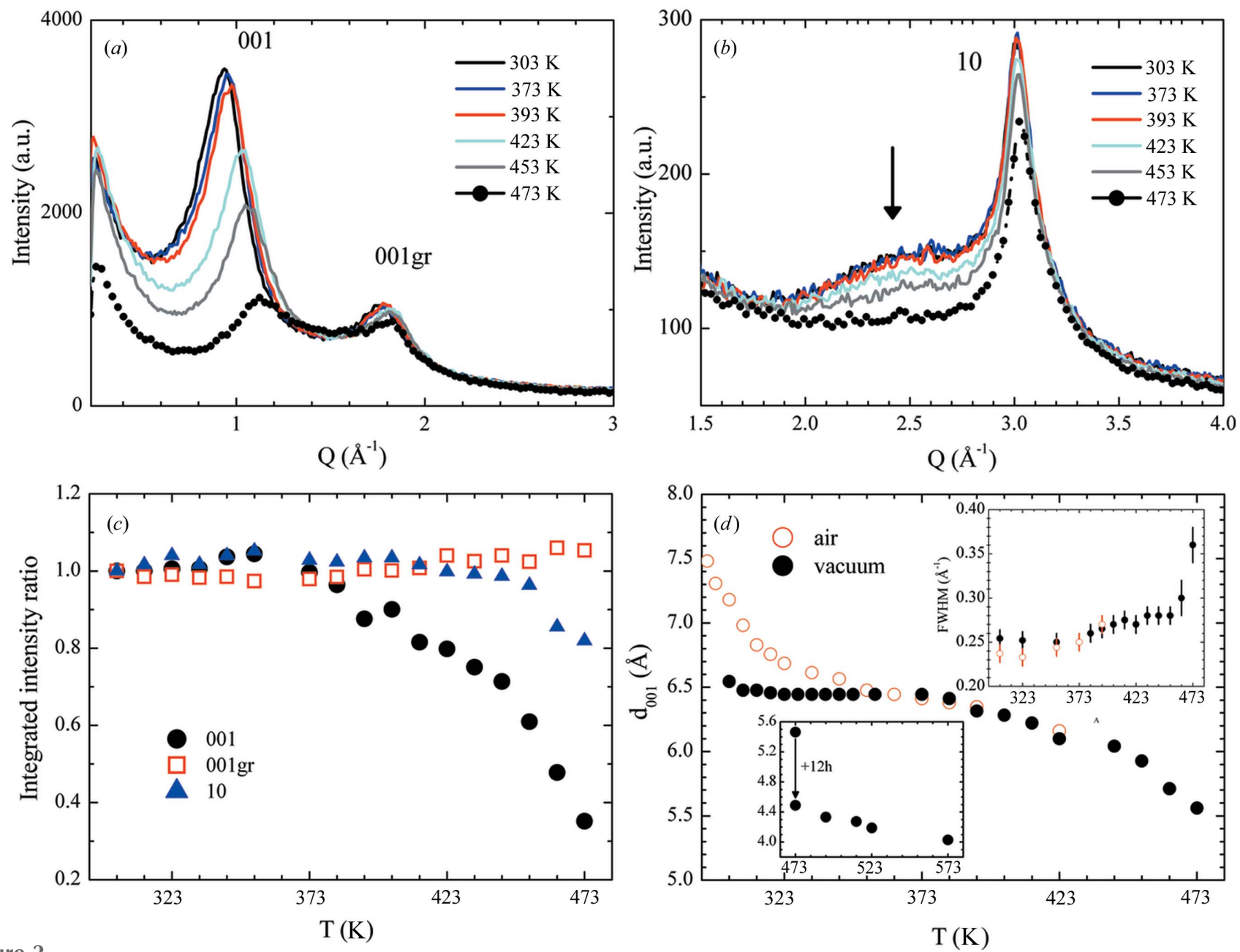

Figure 2

Intensity at different temperatures and under vacuum along $(a)$ the vertical and $(b)$ the horizontal directions in Fig. 1. The black arrow points to the weak isotropic diffuse scattering. (c) Variation of the integrated intensities of the $001,001_{\mathrm{gr}}$ and 10 reflections as a function of temperature. Integration was performed over $Q$ in the vertical and horizontal directions for the 001 and 10 reflections, respectively. (d) Variation of the interlayer distance $d_{001}$ as a function of temperature under vacuum (black filled circles) and in air (red open circles). Bottom inset: variation of the interlayer distance $d_{001}$ in the temperature range [ $473-573 \mathrm{~K}$ ] after a time lapse of $12 \mathrm{~h}$ at $473 \mathrm{~K}$. Top inset: variation of the FWHM of the 001 reflection. 
$001_{\text {gr }}$ peak remain roughly unaffected during thermal treatment, with constant values $Q \simeq 1.78 \AA^{-1}$ and $\mathrm{FWHM} \simeq$ $0.35 \AA^{-1}$.

\subsection{Hydration of GO paper}

GO paper was hydrated under saturated water vapour pressure $(23.8$ mbar at $298 \mathrm{~K})$. Starting from the value $d_{001} \simeq$ $6.6 \AA$ under vacuum, the interlayer distance is expected to expand according to the number of water molecules to be intercalated. The hydration process was followed as a function of time and the corresponding $00 \mathrm{~L}$ intensity diagrams after 1 , 2 and $15 \mathrm{~h}$ from the hydration start are plotted in Fig. 3. The position of the 001 reflection shifts towards smaller scattering wavevectors, while its intensity increases. The peak width becomes narrower, from FWHM $\simeq 0.2 \AA^{-1}$ after $1 \mathrm{~h}$ to $\mathrm{FWHM} \simeq 0.16 \AA^{-1}$ after $2 \mathrm{~h}$. Conversely, the $001_{\text {gr }}$ peak remains unaffected by the hydration with constant values $Q \simeq$ $1.78 \AA^{-1}$ and $\mathrm{FWHM} \simeq 0.35 \AA^{-1}$. After $1 \mathrm{~h}$, the position of the 001 peak corresponds to an interlayer distance of $8.9 \AA$, which expands to $11.5 \AA$ after $2 \mathrm{~h}$, and then changes only slightly to $11.8 \AA$ after $15 \mathrm{~h}$.

Note the appearance of a weak anisotropic scattering bump at $Q \simeq 1.1 \AA^{-1}$ after $2 \mathrm{~h}$ of hydration. Its azimuthal extension is similar to that of the 001 reflection (inset in Fig. 3), which proves that it is related to the GO layer stacking.

\section{Modelling}

As shown experimentally, the presence of intercalated water in multi-layered GO paper dramatically affects the XRS diagram along the $00 L$ direction. Here, a simple structural model is introduced to simulate $00 \mathrm{~L}$ reflections to enable us to discuss, qualitatively, their position and intensity variations depending on the presence of intercalated water, either physisorbed water or bound water. The model is based on

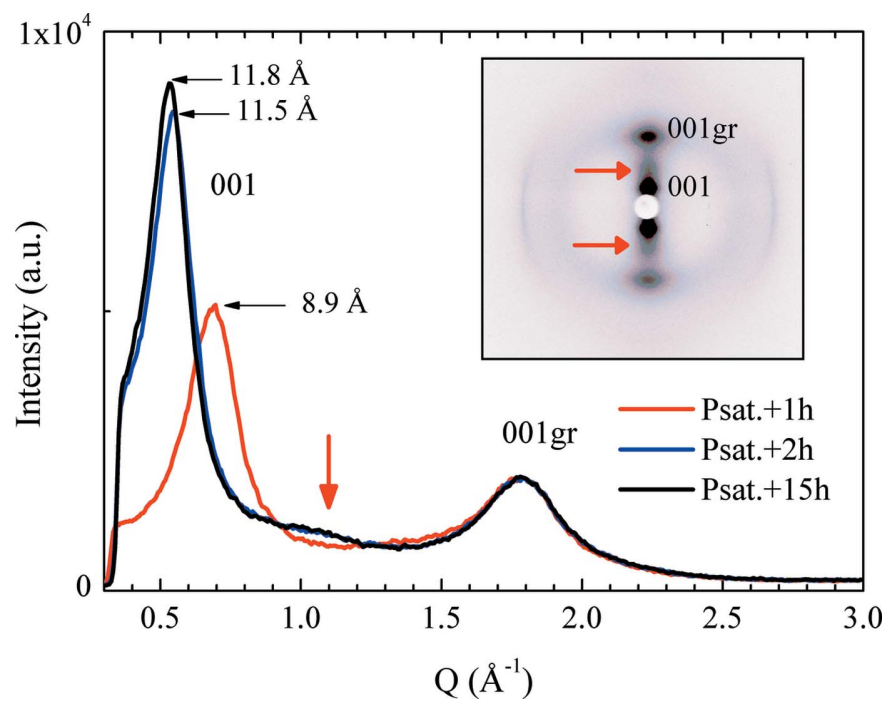

Figure 3

Intensity along the $00 \mathrm{~L}$ direction of GO paper in saturated water vapour atmosphere after 1,2 and $15 \mathrm{~h}$. Inset: XRS image taken after $15 \mathrm{~h}$. Red arrows point out the weak scattering bump at $Q \simeq 1.1 \AA^{-1}$. rigid layers of each atomic or molecular species arranged along the stacking direction. The model allows us to determine the average number of stacked GO layers and the interlayer distance, to investigate the existence of stacking disorder, and to understand the effect of the two different types of intercalated water on the XRS diagram.

\subsection{Layer model}

The model consists of rigid layers of each atomic or molecular species projected onto the stacking axis. Each layer $i$ has its centre of mass located at height $z_{i}$ as sketched in Fig. 4.

The $00 L$ peaks are modelled using the simple expression of the scattering intensity (Warren, 1969)

$$
I_{00 L}^{1}(Q) \propto|F(Q)|^{2} G(Q)
$$

where $G(Q)$ is the interference function depending on the coherence domain of stacking, written, with $N$ the number of layers and $d$ the interlayer distance, as

$$
G(Q)=\frac{1}{N}\left(\frac{\sin N Q d / 2}{\sin Q d / 2}\right)^{2}
$$

The calculated intensity is further corrected by the Lorentzpolarization ( $\mathrm{Lp}$ ) factor adapted to multilayer optics and to a planar detector, $\mathrm{Lp}=\left\{\left[1+\cos ^{2}(2 \theta)\right] / 2\right\} \cos ^{3}(2 \theta)$, with $\theta=$ $\arcsin (Q \lambda / 4 \pi)$.

$F(Q)$ is the structure factor of the layer stacking unit. For a pristine graphene sheet taken as a uniform layer, $F(Q)=$ $f_{\mathrm{C}}(Q)$, with $f_{\mathrm{C}}$ the atomic form factor of carbon. For a GO layer, one has first to consider the different OFGs and hydroxyl and epoxide groups present on the surface of the graphene sheet. By taking hydroxyl $\mathrm{C}-\mathrm{OH}(1.45 \AA)$ and epoxide $\mathrm{C}-\mathrm{O}-\mathrm{C}(1.43 \AA)$ average bond lengths, the heights of the respective layers are $z_{\mathrm{OH}}=1.45$ and $z_{\mathrm{O}}=1.43 \cos 30^{\circ}$ $\left[z_{\mathrm{O}}\right.$ is the projection of the $\mathrm{C}-\mathrm{O}$ bond along the stacking axis, with the angle of the epoxide molecule equal to $60^{\circ}$, as sketched in Fig. 4(b)]. However, in our simple model, we shall consider a single average oxygen layer, located at height $z_{\mathrm{OOH}}=\left(z_{\mathrm{OH}}+z_{\mathrm{O}}\right) / 2=1.344 \AA$, with a weighted layer form factor $f_{\mathrm{OOH}}=\left(f_{\mathrm{O}}+f_{\mathrm{OH}}\right) / 2, f_{\mathrm{O}}$ and $f_{\mathrm{H}}$ being the atomic form factors of oxygen and hydrogen. The same layer is considered on the other side of the graphene plane, at height $-z_{\mathrm{OOH}}$. This simplification is relevant since, to our knowledge, only the ratio O:C can be experimentally determined, from XPS or EELS measurements for instance, varying from 1:1 to $1: 5$ depending on the degree of oxidation of GO (Dreyer et al.,



(a)

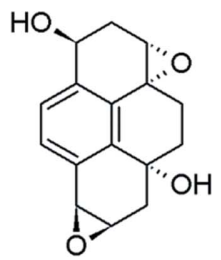

(b)
Figure 4

(a) Scheme of the rigid layer model. (b) Part of a GO layer, with hydroxyl and epoxide groups represented (after Lerf et al., 1998). 
2010; Núñez, 2015; Tararan et al., 2016). Therefore, defining the parameter $p$ as the proportion of total oxygen to carbon (O:C), the structure factor of an anhydrous GO layer can be written as

$$
F(Q)=f_{\mathrm{C}}(Q)+p f_{\mathrm{OOH}}(Q) \cos \left(Q z_{\mathrm{OOH}}\right),
$$

having the carbon layer at $z=0$ and two average oxygen layers, with the proportion of $p / 2$ for each, located at $z_{\mathrm{OOH}}$ and $-z_{\mathrm{OOH}}$, respectively (see Fig. $4 a$ ).

\subsection{Anhydrous GO}

The value of the interlayer distance for anhydrous GO is defined as $d=2 z_{\mathrm{OOH}}+d_{\mathrm{OOH}-\mathrm{OOH}}$. We choose an arbitrary value $d_{\mathrm{OOH}-\mathrm{OOH}}$ equal to $3.4 \AA$, compatible with the van der Waals radius of oxygen $(1.52 \AA)$. In addition, in the absence of OFGs $\left(p=0, z_{\mathrm{OOH}}=0\right)$, substituting this value into the above formula returns the turbostratic graphite's interlayer distance.

According to numerical simulations from equations (3) and (4), the best value $N$ (average number of layers per stack) approximating both the experimental position and the width of the $001 \mathrm{GO}$ reflection under vacuum or at $373 \mathrm{~K}\left(Q_{001} \simeq\right.$ $0.98 \AA^{-1}$ and FWHM $=0.35 \AA^{-1}$ ) where all physisorbed water has been removed, is found to be $N=4$ (see Table S1). Likewise, the number of graphitic layers is determined to be about 4 , with an interlayer distance of $3.45 \AA^{-1}$ (see Table S2).

Simulated XRS diagrams for GO stacking are shown in Fig. 5. Considering a theoretical GO layer stacking without OFGs $(p=0), 00 L$ reflections spread out from $L=1$ to 5 in the $Q$ range $\left[0-6 \AA^{-1}\right]$. Introducing OFGs by varying the $p$ parameter (see Fig. S4), it is possible to extinguish reflections 002 and 003 , as observed experimentally, for $p=0.8$. This value remains in qualitative agreement with the literature. However, the 004 and 005 reflections are still intense in the

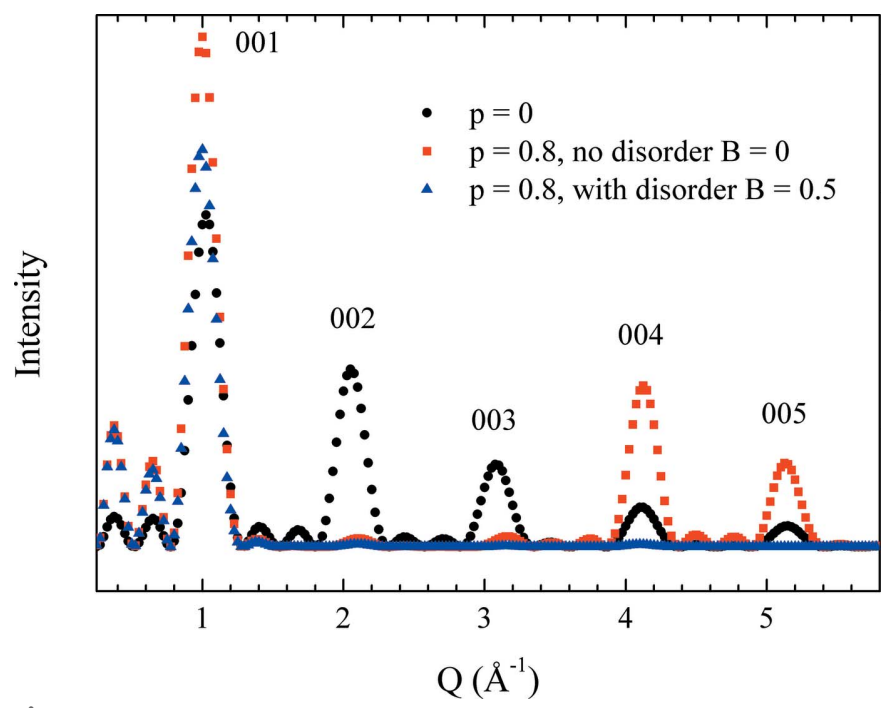

Figure 5

Simulated XRS $00 L$ diagrams of GO with average number of layers per stack $N=4$, for a theoretical GO stacking with no OFGs (black circles), GO stacking (red squares) and GO disordered stacking (blue triangles), with parameter $p=0.8$. simulated diagram. To account for their absence in the experimental diagrams, the introduction of a stacking disorder among GO layers is necessary.

As already stated, GO chemistry is ill-controlled, which yields a non-stoichiometric material with OFGs randomly arranged on graphene planes. Additionally, disorder in the GO layer stacking can have different origins: vacancies or defects within the GO layer inducing local variations of the interlayer distance and layer warping, fluctuations of the layer thickness (layer 'roughness'), and thermal and static layer displacements. All these types of disorder lead differently to the attenuation of $00 L$ reflections and to diffuse scattering between them (Guinier, 1963). However, distinguishing these different types of disorder is out of reach in the present article because of (i) the presence of $00 L_{\mathrm{gr}}(L=1$ and 2) reflections due to graphitic impurities that hinder accurate measurement and analysis of the diffuse scattering and (ii) the absence of higher-order reflections ( $L=2$ and 3 ) beyond the only observed 001 reflection. Therefore, in order to take account of the strong attenuation of $00 L$ reflections $(L=4$ and 5), we shall use a pseudo-Debye-Waller factor that generalizes the stacking disorder in GO, with the scattered intensity expressed as

$$
I_{00 L}^{2}(Q) \propto I_{00 L}^{1} \exp \left(-B^{2} Q^{2}\right)
$$

The parameter $B$ is determined by XRS simulations (Fig. S5). The value $B=0.5 \AA$ strongly attenuates the 004 and 005 reflections, as shown in Fig. 5 and in accordance with the experiments. This result, considered along with the experimental interlayer distance $d_{001}$ values of between 6 and $12 \AA$, reflects the strong disorder present in the GO layers' stacking.

\subsection{Physisorbed water}

Physisorbed water molecules can be added to the model as a rigid layer, the centre of mass of which is located at the position $z_{\mathrm{w}}$ (Fig. $4 a$ ), with $z_{\mathrm{w}}>z_{\mathrm{OOH}}$. The interlayer distance corresponding to the structure becomes $d=2 z_{\mathrm{w}}+d_{\mathrm{w}-\mathrm{w}}, d_{\mathrm{w}-\mathrm{w}}$ being the distance between water layers of neighbouring GO layers, with arbitrary value $d_{\mathrm{w}-\mathrm{w}}=3.4 \AA$.

The structure factor of GO can be written as

$$
\begin{aligned}
F(Q)= & f_{\mathrm{C}}(Q)+p f_{\mathrm{OOH}}(Q) \cos \left(Q z_{\mathrm{OOH}}\right) \\
& +p_{\mathrm{w}_{\mathrm{H}}} f_{\mathrm{H}_{2}}(Q) \cos \left(Q z_{w}\right),
\end{aligned}
$$

where $p_{\mathrm{w}}$ is the ratio of physisorbed water molecules to carbon atoms. The water layers are located at $z_{\mathrm{w}}$ and $-z_{\mathrm{w}}$, with the proportion $p_{\mathrm{w}} / 2$ for each (see Fig. $4 a$ ).

Simulated XRS diagrams as a function of the height $z_{\mathrm{w}}$ of the water layer and of the proportion of water $p_{\mathrm{w}}$ are plotted in Fig. 6. For a given amount of physisorbed water in the structure, here $p_{w}=0.1$, it is shown that the 001 reflection moves towards smaller $Q$ values as the interlayer distance $d$ increases while its intensity increases. The behaviour of the simulated 001 reflection is similar to that of the experimental one (Fig. 3), within the same range of interlayer distances $d$, [7.5-11.4 $\AA$ ]. We note that the FWHM narrows while $d$ increases as measured during the hydration process, with the 
number of stacked layers kept constant $(N=4)$ for all simulated curves. In brief, variations in the position, intensity and FWHM of the 001 reflection are directly connected to the expansion of $d$ due to the intercalation of water in GO stacking, while the diffracting coherent domain remains unchanged with the same number of stacked layers.

An additional peak is present at $Q \simeq 1.1 \AA^{-1}$ in the simulated curve for $d=11.4 \AA$ (Fig. 6), at the same $Q$ value as the scattering bump observed experimentally (Fig. 3). It also appears for the simulated curve with $d=9.4 \AA$, but it is shifted to the position $Q \simeq 1.3 \AA^{-1}$ and weaker in intensity. This additional peak is actually the second-order reflection 002. Nonetheless, it is direct evidence of the presence of intercalated water as the intensity of this peak is related to the amount of physisorbed water. The inset of Fig. 6 shows simulated curves for the interlayer distance $d=11.4 \AA$ as a function of the water proportion $p_{\mathrm{w}}$. On increasing the $p_{\mathrm{w}}$ value up to 0.5 , the intensities of the 001 and 002 reflections strongly decrease, while the 003 reflection rises at position $Q_{003} \simeq 1.65 \AA^{-1}$. The value $p_{\mathrm{w}}=0.1$ is the closest to the experimental case in which the 003 reflection is not observed.

From the value $p_{w}=0.1$, the water weight fraction (\%) can be estimated with the expression $M=p_{\mathrm{w}}\left(m_{\mathrm{H}_{2} \mathrm{O}} \sigma_{\mathrm{H}_{2} \mathrm{O}}\right) /\left(m_{\mathrm{C}} \sigma_{\mathrm{C}}\right)$, $m_{i}$ and $\sigma_{i}$ being the mass of the atom (molecule) $i$ and the respective atomic (molecular) surface density of the layer $\left(m_{\mathrm{H}_{2} \mathrm{O}}=18\right.$ a.u., $m_{\mathrm{C}}=12$ a.u., $\sigma_{\mathrm{C}}=0.38$ atoms $\AA^{-1}, \sigma_{\mathrm{H}_{2} \mathrm{O}} \simeq$ 0.1 molecules $\AA^{-2}$ ). The estimation yields a water weight fraction $M$ of about $4 \%$. We stress that only physisorbed water is considered in this estimated value, excluding bound water intercalated within the GO layer. This estimated value is lower than that obtained by TGA (see supporting information) since the sample was subject to the vacuum outgassing process in our XRS measurements.

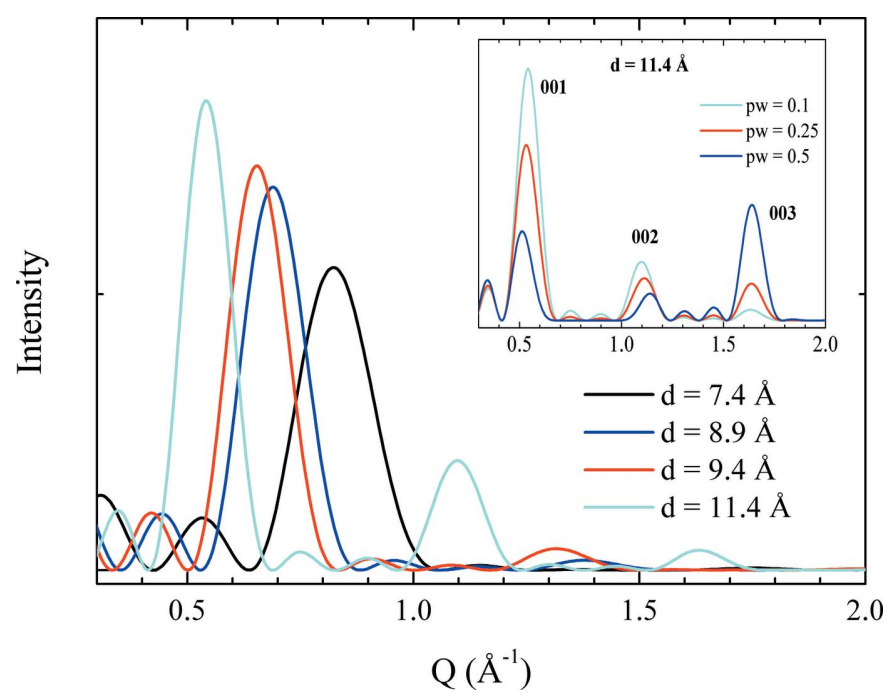

Figure 6

Simulated XRS $00 L$ diagrams of hydrated GO paper with parameters $N=4, p=0.8, p_{\mathrm{w}}=0.1, B=0.5 \AA$ and varying $z_{\mathrm{w}}=2,2.75,3,4 \AA$ [see equation (5)]. Inset: simulated XRS $00 L$ diagrams as a function of the water proportion $p_{\mathrm{w}}$, with interlayer distance $d=11.4 \AA$ and parameters $B=0.5 \AA$.

\subsection{Bound water}

Bound water molecules strongly interact with OFGs and are intercalated within the GO layer. It is thus reasonable to state that the bound water layer is located at a height $z_{\mathrm{bw}}<z_{\mathrm{OOH}}$. The interlayer distance is then kept constant $\left(d=2 z_{\mathrm{OOH}}+\right.$ $\left.d_{\mathrm{OOH}-\mathrm{OOH}}\right)$. Bound water molecules can also possibly be found in holes of the graphene sheet $\left(z_{\mathrm{bw}}=0\right)$. Considering the parameters $p_{\mathrm{h}}$, which defines the proportion of bound water molecules in holes to the total number of bound water molecules, and $p_{\mathrm{bw}}$, the proportion of bound water molecules to carbon atoms, the structure factor can be written as

$$
\begin{aligned}
F(Q)= & f_{\mathrm{C}}(Q)+p f_{\mathrm{OOH}}(Q) \cos \left(Q z_{\mathrm{OOH}}\right) \\
& +p_{\mathrm{bw}} f_{\mathrm{H}_{2} \mathrm{O}}(Q)\left[p_{\mathrm{h}}+\left(1-p_{\mathrm{h}}\right) \cos \left(Q z_{\mathrm{bw}}\right)\right] .
\end{aligned}
$$

The simulations of the XRS $00 L$ diagram in Fig. 7 show that the effect of the presence of bound water is to increase strongly the intensity of the 001 reflection compared to that of anhydrous GO, whenever the bound water layer is located in holes or at a given height $z_{\mathrm{bw}}$ within the GO layer. The proportion $p_{\mathrm{bw}}$ of bound water to carbon was chosen to be 0.25 in order to simulate the single $001 \mathrm{GO}$ reflection (see Fig. S6). Increasing the proportion of water in holes only slightly increases the intensity of the 001 reflection. On the other hand, its intensity is decreased when the height of the bound water layer is progressively increased from 0 to $1 \AA$ while the proportion $p_{\text {bw }}$ is kept constant (Fig. 7 inset). However, it is difficult to go further in the analysis. Anyway, the presence of bound water leads to a more intense 001 reflection when compared to the anhydrous case. Consequently, the removal of bound water contributes to the diminution of the intensity of the 001 reflection observed experimentally above $373 \mathrm{~K}$.

Moreover, the attenuation of the 001 reflection's intensity above $T=373 \mathrm{~K}$ coincides with a progressive decrease of the

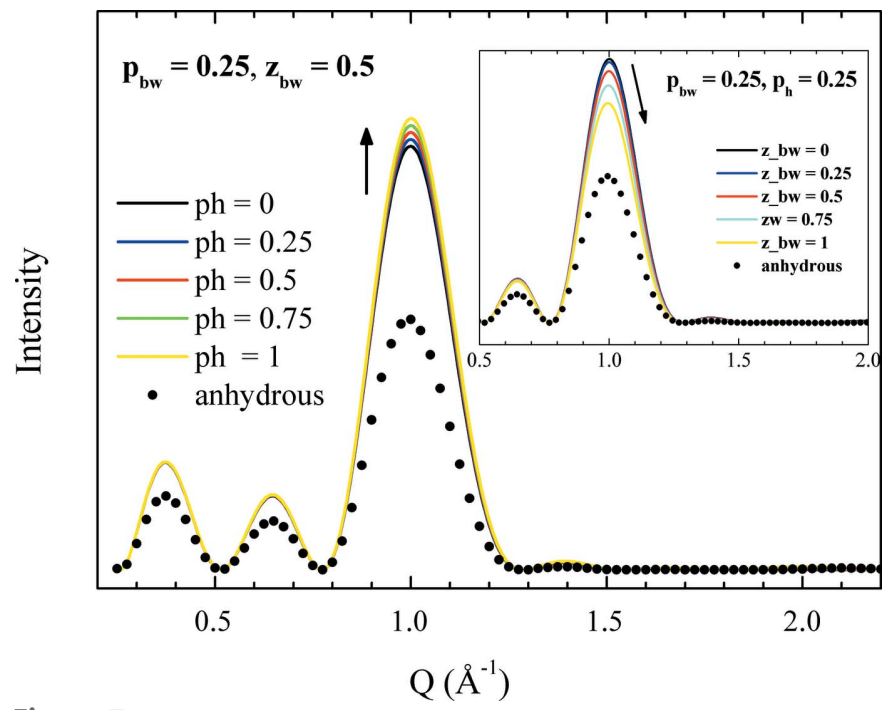

Figure 7

Simulated XRS $00 L$ diagrams as a function of the proportion of bound water in holes $p_{\mathrm{h}}$ to that of a layer centred at $z_{\mathrm{bw}}=0.5 \AA$. Inset: Simulated XRS diagrams as a function of the height $z_{\mathrm{bw}}$, with fixed $p_{\mathrm{h}}=0.25$. All simulations use the parameters $N=4, p=0.8, p_{\mathrm{bw}}=0.25$, $B=0.5 \AA$ 
Table 1

Summarized results from the XRS analysis: temperature ranges with the corresponding values of the interlayer distance related to adsorption/ desorption processes of the different types of water intercalated in the $\mathrm{GO}$ structure.

\begin{tabular}{|c|c|c|c|}
\hline $\begin{array}{l}\text { Temperature } \\
\text { range }(\mathrm{K})\end{array}$ & $\begin{array}{l}\text { Experimental } \\
\text { process }\end{array}$ & $\begin{array}{l}\text { Interlayer } \\
\text { distance } \\
\text { range }(\AA)\end{array}$ & $\begin{array}{l}\text { Physical-chemical } \\
\text { process }\end{array}$ \\
\hline 298 & Hydration & $6.4-11.8$ & $\begin{array}{l}\text { Adsorption of } \\
\text { physisorbed water }\end{array}$ \\
\hline $298-373$ & $\begin{array}{l}\text { Heating or vacuum } \\
\text { degassing }\end{array}$ & $7.5-6.4$ & $\begin{array}{l}\text { Desorption of } \\
\text { physisorbed water }\end{array}$ \\
\hline $373-423$ & Heating & $6.4-6.1$ & $\begin{array}{l}\text { Desorption of } \\
\text { bound water and OFGs }\end{array}$ \\
\hline $423-473$ & Heating & $6.1-5.5$ & Accelerated desorption \\
\hline
\end{tabular}

interlayer distance below $6 \AA$ (Fig. $2 c$ ). This implies that the removal of bound water is necessarily accompanied by the removal of OFGs, leading to a diminution of the interlayer distance $d$. The role of bound water in the chemical modification of the GO layer and the irreversible decomposition of OFGs during the reduction process has been suggested previously (Acik et al., 2010). The chemical decomposition of GO layers as shown in our experimental XRS results (\$3.2) is confirmed by the XPS measurements (see supporting information).

\section{Conclusion}

XRS measurements were performed under vacuum on a multilayered graphene oxide (GO) paper undergoing thermal treatment in the temperature range [298-473 K] as well as a hydration process in a saturated water vapour atmosphere. The respective effects on the XRS pattern of physisorbed water and bound water intercalated in the GO structure have been assessed with the help of a simple structural layer model. The model allows simulation of the main XRS features observed experimentally. Variations of the interlayer distance and intensities of the XRS reflections are qualitatively well described with the help of this schematic model, which is a rather unexpected result for such a complex system. The relationship between the structural information retrieved from XRS analysis and the physical-chemical behaviour of the GO paper is summarized in Table 1. The present article shows that simple modelling of water adsorption effects on XRS diagrams is an effective tool to understand the related physical-chemical phenomena. Such an approach could be extended to water confined within other two-dimensional materials (Li et al., 2015).

\section{Acknowledgements}

EP, SR and PL benefited of a grant from the French Research National Agency under the program Investissements d'Avenir (SEDECO project, ANR-10-LABX-0035: Labex NanoSaclay). AMB and WKM acknowledge funding from the Spanish Ministry MINECO under projects ENE2013-48816-C5-5-R and ENE 2016-79282-C5-1-R with their associated European Regional Development Funds, and from the Government of
Aragon under Consolidated Group programme DGA-T66GCNN and the associated European Social Fund.

\section{References}

Acik, M., Mattevi, C., Gong, C., Lee, G., Cho, K., Chhowalla, M. \& Chabal, Y. J. (2010). ACS Nano, 4, 5861-5868.

Algara-Siller, G., Lehtinen, O., Wang, F. C., Nair, R. R., Kaiser, U., Wu, H. A., Geim, A. K. \& Grigorieva, I. V. (2015). Nature, 519, 443445.

Barroso-Bujans, F., Cerveny, S., Alegría, A. \& Colmenero, J. (2010). Carbon, 48, 3277-3286.

Buchsteiner, A., Lerf, A. \& Pieper, J. (2006). J. Phys. Chem. B, 110, 22328-22338.

Cerveny, S., Barroso-Bujans, F., Alegría, A. \& Colmenero, J. (2010). J. Phys. Chem. C, 114, 2604-2612.

Dreyer, D., Park, S., Bielawski, C. W. \& Ruoff, R. S. (2010). Chem. Soc. Rev. 39, 228-240.

Gao, W., Alemany, L. B., Ci, L. \& Ajayan, P. M. (2009). Nat. Chem. 1, 403-408.

Guinier, A. (1963). X-ray Diffraction in Crystals, Imperfect Crystals and Amorphous Bodies. New York: Dover Publications.

Guo, S., Meshot, E. R., Kuykendall, T., Cabrini, S. \& Fornasiero, F. (2015). Adv. Mater. 27, 5726-5737.

Holt, J. K., Park, H. G., Wang, Y., Stadermann, M., Artyukhin, A. B., Grigoropoulos, C. P., Noy, A. \& Bakajin, O. (2006). Science, 312, 1034-1037.

Huh, S. H. (2011). Physics and Applications of Graphene Experiments, edited by S. Mikhailov, ch. 5. InTech.

Hummers, W. S. Jr \& Offeman, R. E. (1958). J. Am. Chem. Soc. 80, 1339.

Joshi, R. K., Carbone, P., Wang, F. C., Kravets, V. G., Su, Y., Grigorieva, I. V., Wu, H. A., Geim, A. K. \& Nair, R. R. (2014). Science, 343, 752-754.

Kolesnikov, A. I., Zanotti, J.-M., Loong, C.-K., Thiyagarajan, P., Moravsky, A. P., Loutfy, R. O. \& Burnham, C. J. (2004). Phys. Rev. Lett. 93, 035503.

Kyakuno, H., Matsuda, K., Yahiro, H., Inami, Y., Fukuoka, T., Miyata, Y., Yanagi, K., Maniwa, Y., Kataura, H., Saito, T., Yumura, M. \& Iijima, S. (2011). J. Chem. Phys. 134, 244501.

Lerf, A., Buchsteiner, A., Pieper, J., Schöttl, S., Dekany, I., Szabo, T. \& Boehm, H. P. (2006). J. Phys. Chem. Solids, 67, 1106-1110.

Lerf, A., He, H., Forster, M. \& Klinowski, J. (1998). J. Phys. Chem. B, 102, 4477-4482.

Li, Q., Song, J., Besenbacher, F. \& Dong, M. (2015). Acc. Chem. Res. 48, 119-127.

Loh, K. P., Bao, Q., Eda, G. \& Chhowalla, M. (2010). Nat. Chem. 2 , 1015-1024.

Majumder, M., Chopra, N., Andrews, R. \& Hinds, B. J. (2005). Nature, 438, 44.

Mochizuki, K. \& Koga, K. (2015). Proc. Natl Acad. Sci. USA, 112, 8221-8226.

Nair, R. R., Wu, H. A., Jayaram, P. N., Grigorieva, I. V. \& Geim, A. K. (2012). Science, 335, 442-444.

Núñez, J. D. (2015). PhD thesis, University of Zaragoza, Spain.

Núñez, J. D., Benito, A. M., Rouzière, S., Launois, P., Arenal, R., Ajayan, P. M. \& Maser, W. K. (2017). Chem. Sci. https://doi.org/ 10.1039/C7SC00223H.

Pan, Q., Chung, C.-C., He, N., Jones, J. L. \& Gao, W. (2016). J. Phys. Chem. C, 120, 14984-14990.

Pei, S. \& Cheng, H. M. (2012). Carbon, 50, 3210-3228.

Rezania, B., Severin, N., Talyzin, A. \& Rabe, J. P. (2014). Nano Lett. 14, 3993-3998.

Stankovich, S., Dikin, D. A., Piner, R. D., Kohlhaas, K. A., Kleinhammes, A., Jia, Y., Wu, Y., Nguyen, S. T. \& Ruoff, R. S. (2007). Carbon, 45, 1558-1565.

Talyzin, A. V., Hausmaninger, T., You, S. \& Szabo, T. (2014). Nanoscale, 6, 272. 
Talyzin, A. V., Luzan, S. M., Szabó, T., Chernyshev, D. \& Dmitriev, V. (2011). Carbon, 49, 1894-1899.

Tararan, A., Zobelli, A., Benito, A. M., Maser, W. K. \& Stéphan, O. (2016). Chem. Mater. 28, 3741-3748.

Vallés, C., David Núñez, J., Benito, A. M. \& Maser, W. K. (2012). Carbon, 50, 835-844.
Warren, B. E. (1969). X-ray Diffraction. New York: Dover Publications.

You, S., Sundqvist, B. \& Talyzin, A. V. (2013). ACS Nano, 7, 13951399.

Zhu, J., Andres, C. M., Xu, J., Ramamoorthy, A., Tsotsis, T. \& Kotov, N. A. (2012). ACS Nano, 6, 8357-8365. 